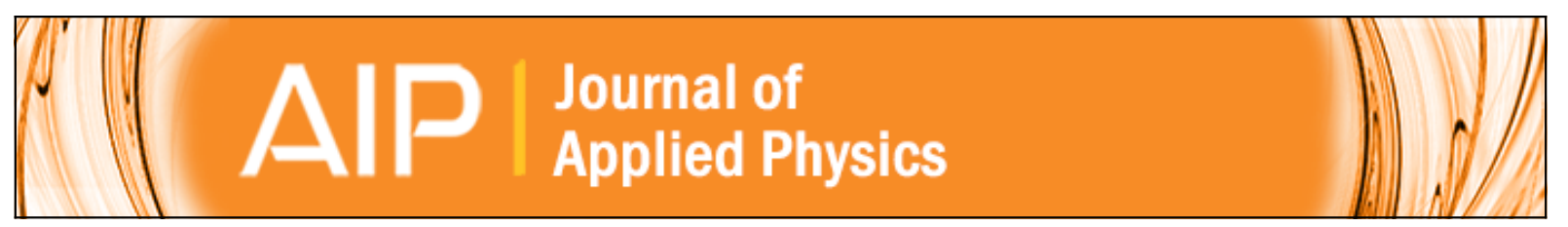

Photopolymerizable organically modified holographic glass with enhanced thickness for spectral filters

A. V. Velasco, M. L. Calvo, and P. Cheben

Citation: Journal of Applied Physics 113, 033101 (2013); doi: 10.1063/1.4775787

View online: http://dx.doi.org/10.1063/1.4775787

View Table of Contents: http://scitation.aip.org/content/aip/journal/jap/113/3?ver=pdfcov

Published by the AIP Publishing

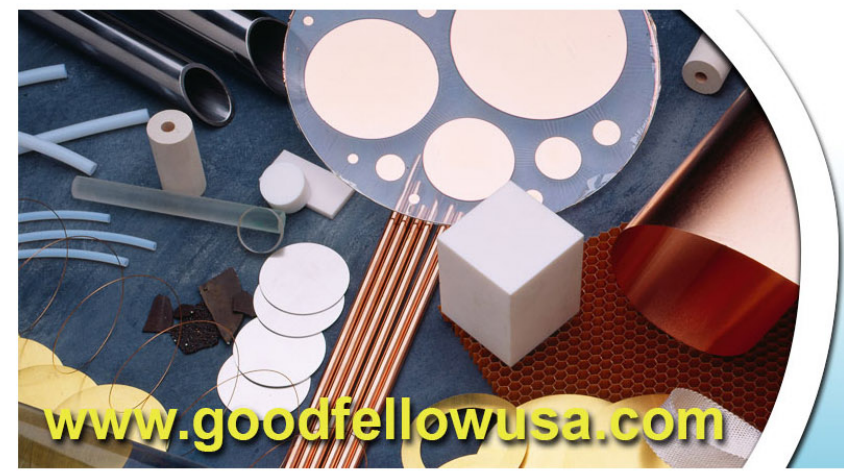

BODIFEl|OII

metals • ceramics • polymers composites • compounds • glasses

Save $5 \% \cdot$ Buy online 70,000 products $\cdot$ Fast shipping 


\title{
Photopolymerizable organically modified holographic glass with enhanced thickness for spectral filters
}

\author{
A. V. Velasco, ${ }^{1}$ M. L. Calvo, ${ }^{1}$ and P. Cheben ${ }^{2}$ \\ ${ }^{1}$ Department of Optics, Faculty of Physics, Complutense University of Madrid, 28040 Madrid, Spain \\ ${ }^{2}$ National Research Council Canada, Ottawa, Ontario K1A OR6, Canada
}

(Received 7 November 2012; accepted 21 December 2012; published online 15 January 2013)

\begin{abstract}
A novel formulation and synthesis method to overcome the thickness limitations in samples of photopolymerizable glasses with high refractive index species is presented. The reported method allows the recording of volume holographic diffraction gratings in samples of $\sim 500 \mu \mathrm{m}$ thickness with a high optical quality and low scattering. Holographic grating recording is performed in a single coherent light exposure step, resulting in volume gratings of high optical quality. A holographic notch filter implemented in a $500 \mu \mathrm{m}$ thick photopolymerizable glass with a spectral bandwidth below $0.3 \mathrm{~nm}$ and an excellent filter extinction ratio of $<-27 \mathrm{~dB}$ is also demonstrated. (C) 2013 American Institute of Physics. [http://dx.doi.org/10.1063/1.4775787]
\end{abstract}

\section{INTRODUCTION}

Holographic filters are fundamental components used in a variety of applications, such as microscopy, ${ }^{1}$ pattern recognition, ${ }^{2,3}$ beam transformation, ${ }^{4}$ chemical spectrum identification, ${ }^{5}$ and general narrowband filtering, ${ }^{6}$ to name a few. In particular, holographic recording materials are ideal candidates for implementing narrow-band notch filters based on Bragg diffraction gratings, ${ }^{7,8}$ often used for Rayleigh line suppression in Raman spectroscopy. ${ }^{9-11}$ For the development of high-performance filters with a narrow stop-band and a high extinction (central wavelength rejection) ratio, a holographic medium with a high dynamic range, superior optical quality, and large thickness is required. ${ }^{12}$

Photopolymerizable materials have shown excellent performance in terms of dynamic range and optical quality. ${ }^{13,14}$ However, organic polymeric binders used in holographic polymers are typically limited in thickness to less than a few hundred micrometers. Also, dimensional changes induced by light exposure (material shrinkage) or temperature variations can distort the hologram and alter the designed spectral response of the filter.

A new class of holographic material based on organically modified sol-gel glass was developed by Cheben et al. ${ }^{15,16}$ The material is an organic-inorganic photosensitive glass comprising a silicate sol-gel matrix which incorporates monomeric and photoinitiator species. When exposed to an interference pattern at an actinic wavelength, the photoinitiator is activated and the polymerization of the free monomers takes place. Additionally, a diffusion of monomer takes place from the dark towards the illuminated regions where the monomer concentration is depleted. ${ }^{15}$ The refractive index modulation $\Delta n$ of up to $5.6 \times 10^{-3}$ and diffraction efficiencies close to $100 \%$ were reported for this type of material. ${ }^{16}$ The refractive index modulation was subsequently enhanced to $\sim 0.01$ by incorporating a zirconium-based high refractive index species (HRIS) to the photopolymerizable glass. ${ }^{17}$ Zirconium isopropoxide $\mathrm{Zr}\left(\mathrm{O}^{\mathrm{i}} \mathrm{Pr}\right)_{4}$ high index species was introduced at a molecular level to minimize scattering loss. The incorporation of $\mathrm{ZrO}_{2}$ nanoparticles to polymeric films has also been demonstrated to increase material dynamic rage. ${ }^{18}$ Photopolymerizable glasses have also proven to withstand a high pulsed laser energy without degradation of the recorded diffraction gratings. ${ }^{19}$ The high dynamic range and optical quality of photopolymerizable glasses make them candidates for volume holographic recoding. ${ }^{20-22}$ However, in order to implement holographic filters with high spectral selectivity, a photopolymerizable holographic recording material with thickness of the order of $500 \mu \mathrm{m}$ or more is required.

In this paper, we present a new formulation and synthesis method for a photopolymerizable glass incorporating HRIS that allows the fabrication of samples with typical thickness of about $500 \mu \mathrm{m}$, as required for applications in holographic filters with high spectral selectivity. The dependence of the photomaterial performance on sample thickness is analyzed, including the angular selectivity, dark diffusion, scattering, and optical quality. A narrowband notch filter implemented in a 500- $\mu$ m-thick photopolymerizable glass is presented.

The paper is organized as follows: Sec. II presents the novel formulation and synthesis procedure for preparation of samples with increased thickness. Section III is dedicated to the experimental characterization of the fabricated samples. In particular, characteristics of samples with thickness ranging from 50 to $500 \mu \mathrm{m}$ are compared and the relation between sample thickness and holographic performance is analyzed. In Sec. IV, we present a notch filter implemented in a 500- $\mu$ m-thick photopolymerizable glass. Discussions and conclusions are presented in Sec. V.

\section{SAMPLES PREPARATION}

In order to overcome the thickness limitation of the photopolymerizable glass samples with HRIS, some modifications were introduced to the synthesis method previously disclosed. ${ }^{17}$ Here, we summarize the modified method, with more details described in the Appendix. First, the concentration of HRIS in the samples was reduced compared to Ref. 17 to minimize tendency of cracking in thick samples. 
In order to minimize the consequential decrease in dynamic range $(\Delta n)$ and prolonged gelation times, we chose the following molarity of $\mathrm{Zr}\left(\mathrm{O}^{\mathrm{i}} \mathrm{Pr}\right)_{4} \cdot{ }^{\mathrm{i}} \mathrm{PrOH}: 0.75 \mathrm{mmol}$ and $\mathrm{MA}$ : $3.5 \mathrm{mmol}$, while maintaining the other component concentrations as disclosed in Ref. 17.

Second, instead of the microscope slides, we used Petri dishes to contain an increased amount of the sol-gel solution. Once the solution was cast on the dish, each sample was individually sealed using wax paper and stored in an oven with a controlled temperature of $40^{\circ} \mathrm{C}$ and a relative humidity of $25 \%$. Samples with thicknesses ranging from 50 to $500 \mu \mathrm{m}$ were prepared. The process lasted up to 14 days for the thickest samples. As the gelation evolved, oxygen flow was progressive allowed to the sample by making additional small holes in the wax paper sealing, in order to avoid abrupt changes in the environmental conditions which could result in cracking and surface deterioration.

\section{SAMPLES HOLOGRAPHIC CHARACTERIZATION}

\section{A. Holographic recording and characterization setup}

Transmission volume phase holographic gratings (VPHG) were recorded in the photopolymerizable glass samples by interference of two coherent $s$-polarized beams using a Mach-Zehnder interferometer with a single-frequency solid state laser at a wavelength of $532 \mathrm{~nm}$ (Oxxius $532 \mathrm{~S}$ ). Beams with equal intensities of $7.5 \mathrm{~mW} / \mathrm{cm}^{2}$ were used for maximal visibility of the interference fringes. A spatial frequency of 500 lines $/ \mathrm{mm}$ was obtained using incidence angles of $\pm 7.9^{\circ}$ from the sample normal. Samples were stabilized by exposure to incoherent light after the holographic gratings recording.

A non-actinic probe beam from a He-Ne laser at $632.8 \mathrm{~nm}$ wavelength with an output power of $0.5 \mathrm{~mW}$ (Newport ULMTILT) was used for real-time monitoring of the evolution of the grating formation. Angular selectivity of the recorded gratings was characterized by placing the sample on a high-precision rotation platform and measuring the $1 \mathrm{st}$ Bragg diffraction order using the He-Ne laser. Refractive index modulation values were obtained by fitting the measured angular selectivity curve to the theoretical expression from Kogelnik's coupled wave theory for light diffraction by dielectric transmission volume gratings ${ }^{23}$

$$
\Delta n=2 n_{0} \operatorname{Cos}\left(\vartheta_{0}\right) \operatorname{Sin}\left(\vartheta_{0}\right) T \frac{\operatorname{ArcSin}(\sqrt{\eta})}{\sqrt{\pi^{2}-(\operatorname{ArcSin}(\sqrt{\eta}))^{2}}},
$$

where $n_{o}$ is the refractive index of the photomaterial prior to holographic exposure, $\theta_{0}$ is the Bragg angle, $T$ is the sample thickness, and $\eta$ is the diffraction efficiency. The latter was calculated as $\eta=P_{-1} / P_{0}$, where $P_{-1}$ is the power in the -1 st diffraction order and $P_{0}$ is the power in the probe beam incident on the sample.

\section{B. Diffraction efficiency and angular selectivity}

Figure 1 shows the angular selectivity curves of three diffraction gratings with thicknesses $150 \mu \mathrm{m}, 260 \mu \mathrm{m}$, and
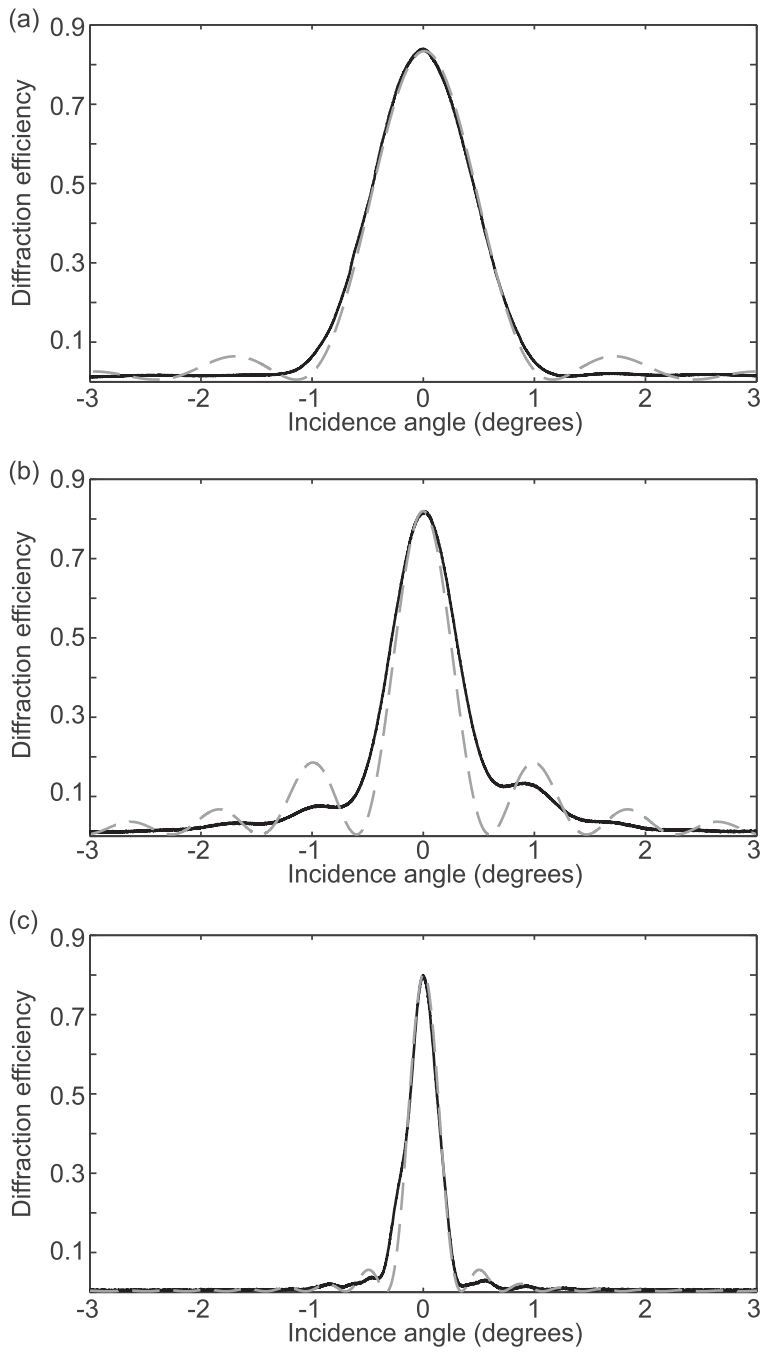

FIG. 1. Measured angular selectivity (solid) and theoretical fitting (dashed) of volume holographic gratings in a photopolymerizable glass of thickness: (a) $150 \mu \mathrm{m}$, (b) $260 \mu \mathrm{m}$, and (c) $500 \mu \mathrm{m}$. Grating of spatial frequency 500 lines/mm were recorded with a solid-state laser at $532 \mathrm{~nm}$ and monitored with a non-actinic He-Ne laser at $632 \mathrm{~nm}$. Incidence angles are measured from the Bragg angle.

$500 \mu \mathrm{m}$. It can be observed that the angular selectivity curves fit well to the theoretical response near Bragg's angle, confirming that the gratings were efficiently recorded in the entire volume of the sample. The increase of angular selectivity with sample thickness is observed, as expected. For the $500 \mu \mathrm{m}$ thick grating, a $50 \%(3 \mathrm{~dB})$ roll-off is achieved for a $0.15^{\circ}$ detuning from the Bragg angle $\left(9.4^{\circ}\right.$ for the read-out laser at $632 \mathrm{~nm}$ ). The ratio between the maximum diffraction efficiency and the intensity of secondary lobes is only 0.04 $(-14 \mathrm{~dB})$.

In Fig. 1, the angular selectivity curve for the $500 \mu \mathrm{m}$ diffraction grating corresponds to a diffraction efficiency of $80 \%$ at Bragg's angle. This specific value is limited by the overexposure, and higher diffraction efficiency values close to $100 \%$ theoretical limit can be achieved. This is shown in Figure 2, where the evolution of the diffraction efficiency is plotted after the recording pulse with a maximum diffraction efficiency of $99 \%$ approximately $7 \mathrm{~s}$ following the recording pulse. As a result of the high dynamic range of the sample, 


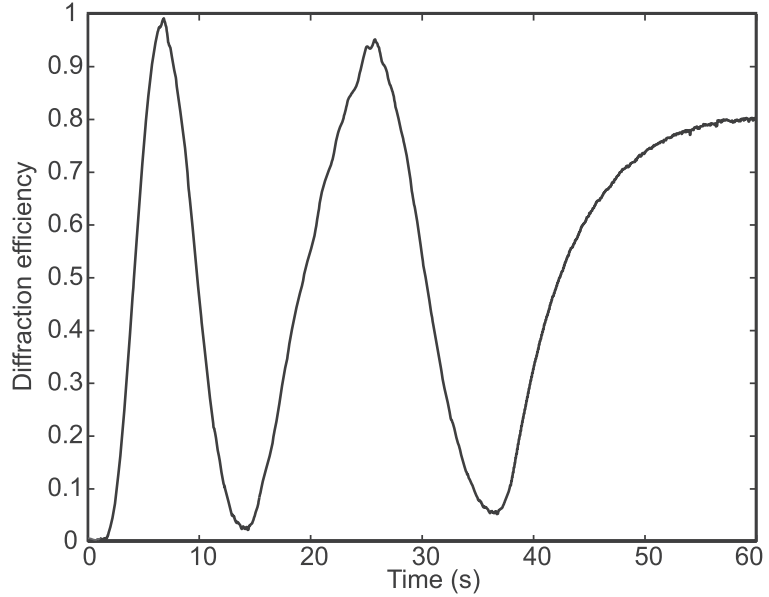

FIG. 2. Evolution of the diffraction efficiency at Bragg's angle of a $500 \mu \mathrm{m}$ thick holographic grating after a single recording pulse of $15 \mathrm{~mJ} / \mathrm{cm}^{2}$ at $532 \mathrm{~nm}$ wavelength. Grating evolution is monitored with a non-actinic beam from a He-Ne laser of $632 \mathrm{~nm}$ wavelength.

the refractive index modulation keeps increasing after this point, resulting in the overmodulation and a corresponding decrease in diffraction efficiency. The evolution of the grating formation is further discussed in Sec. III C.

Since diffraction efficiencies close to $100 \%$ can be readily obtained, and given the high thickness and dynamic range of the synthesized samples, highly selective holographic filters, both angularly and spectrally, can be implemented in our photopolymerizable glass. Additionally, the recording of gratings with spatial frequencies exceeding 4000 lines/mm was demonstrated in our material. ${ }^{24}$ This provides an additional degree of freedom to further increase the spectral and angular selectivity of our grating filters, if demanded by specific applications.

\section{Dark diffusion and sensitivity}

As a result of the polymerization of the monomer species in the illuminated regions, spatial nonuniformities in the distribution of the monomer and HRIS are induced during the exposure in our material. These concentration gradients trigger a migration of the monomer and HRIS from monomer/HRIS rich (dark fringes) regions to monomer/HRIS depleted regions (bright fringes) of the interference pattern. As the diffused species are polymerized, the refractive index modulation of the grating is further increased. The diffusion, which occurs in the absence of light, and the subsequent $\Delta n$ evolution, is referred to as "dark diffusion," and stops when the composition reaches its chemical equilibrium. In the case of the photopolymerizable glass with HRIS, dark diffusion mechanism can be modeled using the weighted sum of two exponential functions ${ }^{25,26}$

$$
\Delta n(t)=C_{M}\left[1-\exp \left(-t / \tau_{M}\right)\right]+C_{Z r}\left[1-\exp \left(-t / \tau_{Z r}\right)\right],
$$

where exponential weight factors, $C_{M}$ and $C_{Z r}$, account for the partial contributions of monomer and $\mathrm{Zr}$-based HRIS components, respectively, to the overall $\Delta n$ value. The diffusion time constants $\tau_{M}$ and $\tau_{Z r}$, account for the different diffusion speeds of the two respective species, namely $\tau_{M}<\tau_{Z}$.
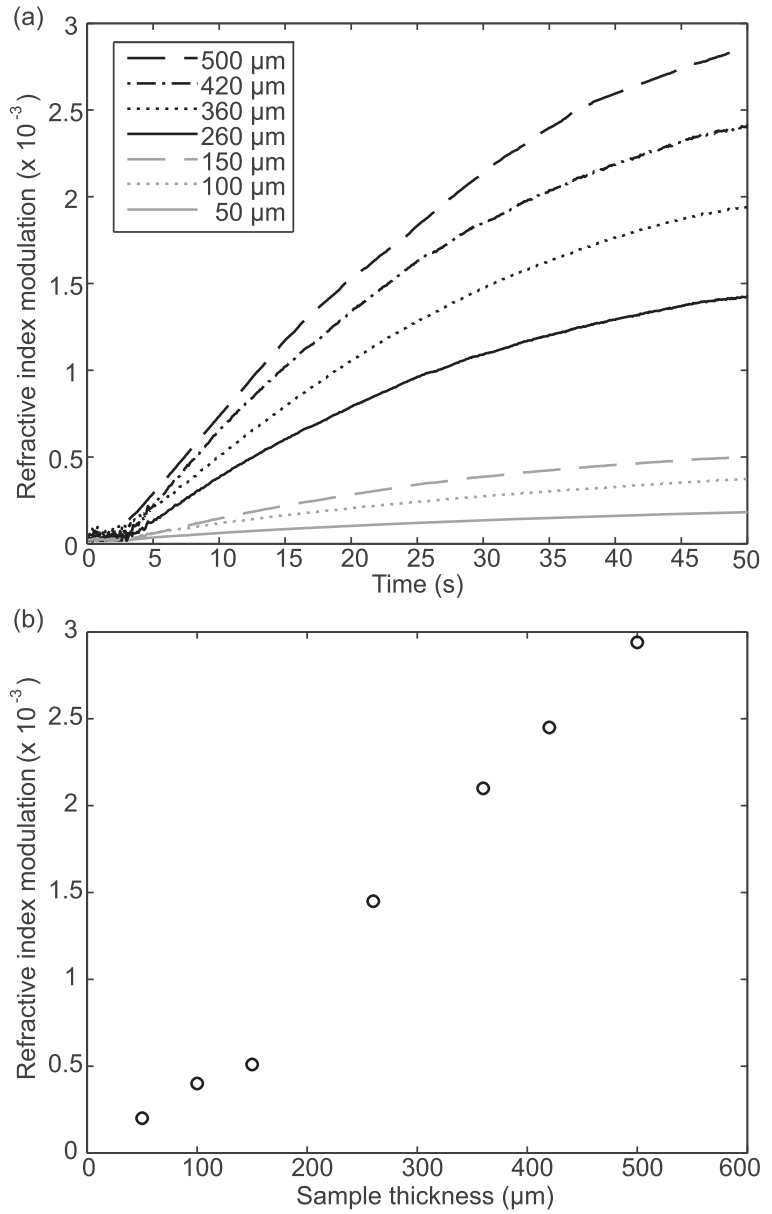

FIG. 3. (a) Measurement of dark diffusion of monomer and HRIS species for sample of different thicknesses. $1 \mathrm{~s}$ pulse at $532 \mathrm{~nm}$ and $15 \mathrm{~mW} / \mathrm{cm}^{2}$ flux was used for the recording of gratings with spatial frequency of 500 lines/ mm. $\Delta n$ was measured with a He-Ne laser at $632 \mathrm{~nm}$. (b) Measured refractive index modulation corresponding to a $1 \mathrm{~s}$ recording pulse of $15 \mathrm{~mW} / \mathrm{cm}^{2}$.

This double-exponential behaviour is observed in Fig. 3(a), which shows the temporal evolution of $\Delta n$ after a recording pulse for several gratings with thicknesses ranging from 50 to $500 \mu \mathrm{m}$. The samples show a high sensitivity, which allows diffraction gratings to be recorded with a single pulse of $1 \mathrm{~s}$ and $15 \mathrm{~mW} / \mathrm{cm}^{2}$. An induction period of 2 to $3 \mathrm{~s}$ with a comparatively slow response is followed by a fast evolution with $\Delta n$ increasing as in Eq. (2). The curves slopes are proportional to the $\Delta n$ resulting from a single exposure at a given energy. This parameter increases progressively with the thickness of the sample, allowing to reach high refractive index modulations with low exposure light powers, as it is shown in Fig. 3(b).

Time constants $\tau_{M}$ and $\tau_{Z r}$ are found to be similar for the analyzed samples, as the speed of the diffusion is determined by the composition of the glass matrix with little influence of the sample thickness. By fitting the experimental curves to Eq. (2), average values of $\tau_{M}=5.1 \pm 0.8 \mathrm{~s}$ and $\tau_{Z r}=25.7 \pm 1.2 \mathrm{~s}$ were obtained, showing a stable dark diffusion behavior in the analyzed thickness range (50 to $500 \mu \mathrm{m})$. The measured diffusion time values are also in good agreement with previously published results for photopolymerizable glasses incorporating HRIS. ${ }^{25,26}$ 


\section{Scattering and optical quality}

The samples under investigation presented a high optical quality with low scattering. Residual noise grating formation angular selectivity measurements are presented in Fig. 4, with a negligible scattering penalty as the sample thickness increases. In these measurements, the build-up of a noise grating would be shown as a dip in diffraction efficiency near Bragg resonance, but no such dip is observed for our samples, indicating high optical quality and low scattering. Furthermore, diffraction efficiency variations in the analysed angular range are within a $1 \%$ range, even for the thickest samples, demonstrating the low scattering of the samples.

Figure 5 shows the He-Ne readout beam intensity distribution in (a) free space, (b) transmitted through a $500 \mu \mathrm{m}$ thick unexposed photopolymerizable glass; (c) transmitted through the same $500 \mu \mathrm{m}$ thick photopolymerizable glass after exposure to $15 \mathrm{~mW} / \mathrm{cm}^{2} 1$-s pulse. Strong speckle is initially observed (Fig. 5(c)), caused by light scattering at sample surface irregularities. We mitigated the surface scattering by using a refractive index matching liquid (Norland Index Matching Liquid 150, $n=1.52$ ), as it is shown in Fig. $5(d)$, achieving a good beam quality.

\section{HOLOGRAPHIC NOTCH FILTER}

The available thickness range of our photopolymerizable glasses allows the implementation of high-quality filters for various applications. As an example, a holographic notch filter was implemented in a $510-\mu \mathrm{m}$-thick sample, which is the upper thickness limit that was achieved with the described synthesis method without resulting in fractures or uneven gelation. Samples with thickness in the $0.5-1 \mathrm{~cm}$ range could likely be achieved by judiciously tailoring the preparation process and by reducing their HRIS concentration. The filter was recorded by interference of two coherent $s$-polarized beams from a solid-state laser at $532 \mathrm{~nm}$ with an incidence angle of $48.1^{\circ}$, yielding a grating spatial frequency of 2800 lines $/ \mathrm{mm}$. A single pulse exposure $\left(3 \mathrm{~mJ} / \mathrm{cm}^{2}\right.$ flux, $1 \mathrm{~s}$ duration) was used to achieve maximal diffraction

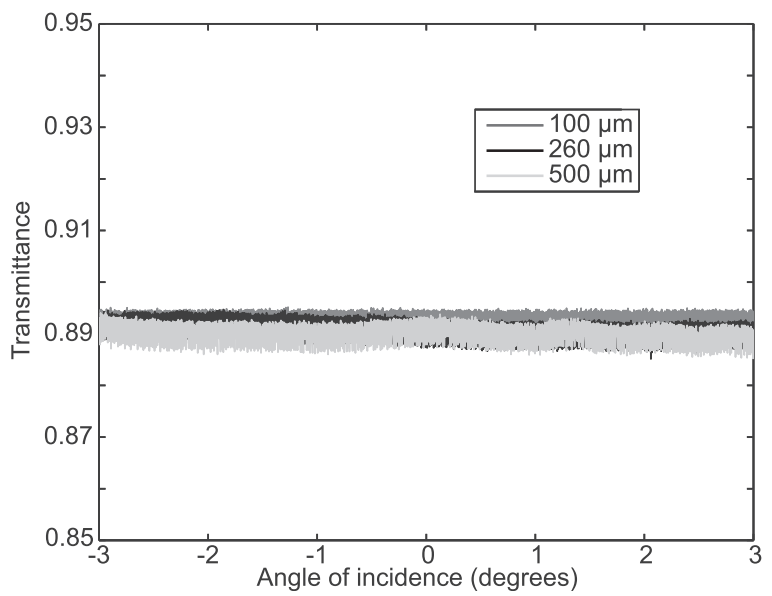

FIG. 4. Noise grating angular selectivity measurements for samples of different thicknesses. The samples were exposed to a coherent $s$-polarized single beam of $532 \mathrm{~nm}$ wavelength and $15 \mathrm{~mW} / \mathrm{cm}^{2}$ flux for $1 \mathrm{~s}$ and read-out by a non-actinic $\mathrm{He}-\mathrm{Ne}$ laser at $632 \mathrm{~nm}$. (a)

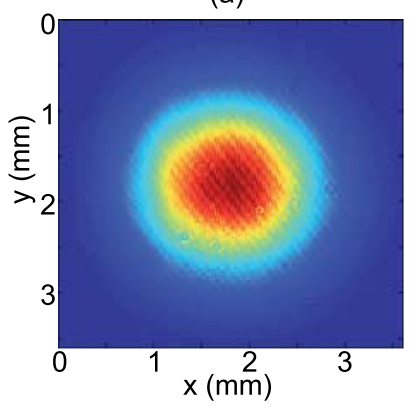

(c)

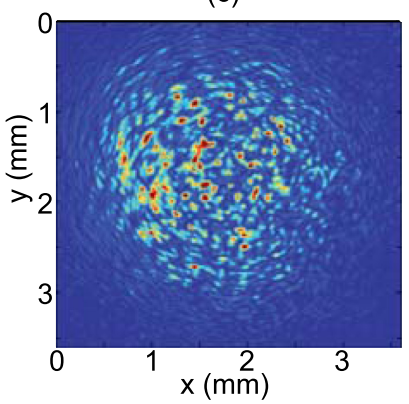

(b)

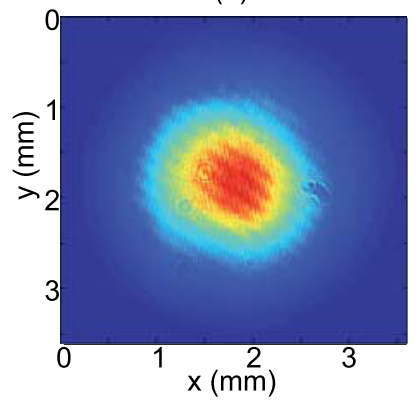

(d)

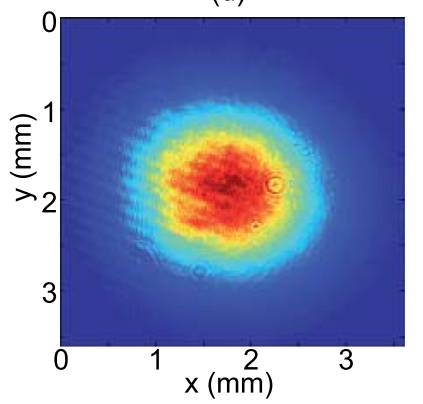

FIG. 5. Beam profiles of a He-Ne laser at $632 \mathrm{~nm}$ (a) in free space; (b) transmitted through a $500 \mu \mathrm{m}$ thick unexposed photopolymerizable glass; (c) transmitted through the same $500 \mu \mathrm{m}$ thick photopolymerizable glass after exposure to $15 \mathrm{~mW} / \mathrm{cm}^{2} 1$-s pulse; (d) as in (c) but after applying a refractive index matching liquid to mitigate surface scattering. All images were taken with a Spiricon digital camera (Ophir) with a pixel size of $4.4 \mu \mathrm{m} \times 4.4 \mu \mathrm{m}$.

efficiency and a high quality grating. Figure 6 shows the experimental measurement of the spectral response of the filter, characterized using a He-Ne laser at $632.8 \mathrm{~nm}$ with incidence angles centred at the Bragg angle of $62.3^{\circ}$. A $-3 \mathrm{~dB}$ bandwidth of $0.3 \mathrm{~nm}$ is achieved, with a maximum suppression of $-27.5 \mathrm{~dB}$ and a flat response for off-Bragg wavelengths with minimal ripple $(<-0.1 \mathrm{~dB})$. The central wavelength of the device can be readily tuned either by modifying the operational angle of incidence or by adjusting the inter-beam during the recording of the filter.

It is obvious that the same device can also be used as a passband filter for the diffracted beam, with the same bandwidth as the notch filter for the transmitted beam. An

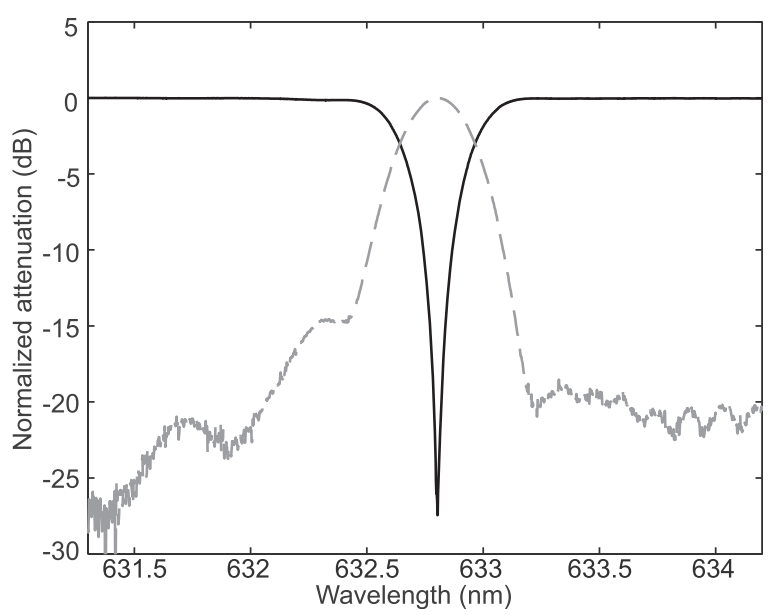

FIG. 6. Holographic filter spectral response for the transmitted beam (black, notch filter) and the diffracted beam (dashed grey, bandpass filter). 
extinction ratio exceeding $-20 \mathrm{~dB}$ is achieved, with sidelobes level of $-15 \mathrm{~dB}$ (Fig. 6, grey dashed curve). The limiting factor which determines this sidelobe level is the theoretical angular selectivity curve of a diffraction grating recorded by interference of two plane wavefronts, as defined by Kogelnik's expression. ${ }^{23}$ Sidelobe level could be potentially optimized by judiciously tailoring the shape of the recording beams with a spatial modulator. Absorption losses are negligible, with the main loss factor being reflections at the facets of the device ( $\sim 4 \%$ per facet for normal incidence), which if required can be compensated using an antireflective coating. Overall, the filter presents a very good optical quality and low scattering for both transmitted and diffracted beams, similar to those shown in Sec. III.

\section{CONCLUSIONS}

We reported a modified composition and method for developing photopolymerizable glasses incorporating HRIS with large thickness $(\sim 500 \mu \mathrm{m})$. Fabricated samples have shown low scattering, and high diffraction efficiency and dynamic range, allowing to tailor holographic filters with high optical quality and selectivity, both angularly and spectrally. The high sensitivity of the photomaterial with HRIS and enhanced thickness allows to record the filter with a single short light pulse. The grating is formed after exposure by internal diffusion of the monomeric species and HRIS inside the glass matrix, thus preventing surface roughness to be transmitted to the grating by subsequent light pulses and reducing the resulting scattering. A high-quality narrowband notch filter has been fabricated with the disclosed method, demonstrating the potential applications of these photopolymerizable glasses for various purposes such as as spectrometry, microscopy, and optical instrumentation, including high power instrumentation due to their high laser damage threshold.

\section{ACKNOWLEDGMENTS}

We are indebted to F. Del Monte (Institute for Materials Science, Spanish Research Council) for helpful discussions. Financial support from the Spanish Ministry of Science and Innovation (MICINN) under Grant Nos. TEC2008-04105 and TEC2011-23629 is acknowledged.

\section{APPENDIX: SAMPLE PREPARATION}

The chemical procedure for the synthesis of the photopolymerizable glass incorporating HRIS was reported in detail in Ref. 17; here we summarize the basic steps.

The silica sol is prepared by acid hydrolysis of glycidoxypropyltrimethoxysilane (GPTMS) and tetraethylorthosilicate (TEOS). The molar ratio between GPTMS and TEOS is selected to minimize the shrinkage of the photopolymerizable glasses after light exposure. ${ }^{27}$ Molarities of the hydrochloric acid solution are also selected to ensure all water is consumed during the hydrolysis. After $10 \mathrm{~min}$ of vigorous stirring, a solution of IRGAGURE-784 photosensitizer in Phenoxyethyl acrylate (POEA) is added. A solution of zirconium isopropoxide isopropanol complex in methacrylic acid is added to the mixture after an additional $10 \mathrm{~min}$. The resulting solution is filtered with a $0.2 \mu \mathrm{m}$ millipore filter.

Films of thickness up to $500 \mu \mathrm{m}$ were obtained by casting the filtered solution on glass Petri dishes. Samples were sealed with wax paper and left to dry in the dark at a controlled temperature of $40^{\circ} \mathrm{C}$ for 14 days prior to hologram recording. Oxygen flow to the sample was progressively increased by performing small holes in the wax paper sealing. A stable relative humidity of $25 \%$ was maintained during the gelation process.

${ }^{1}$ G. Barbastathis, M. Balberg, and D. J. Brady, Opt. Lett. 24, 811 (1999).

${ }^{2}$ H. Fujii, S. P. Almeida, and J. E. Dowling, Appl. Opt. 19, 1190 (1980).

${ }^{3}$ M. Fleisher, U. Mahlab, and J. Shamir, Appl. Opt. 29, 2091 (1990).

${ }^{4}$ M. Quintanilla and A. M. de Frutos, Appl. Opt. 20, 879 (1981).

${ }^{5}$ L. Cao and C. Gu, Appl. Opt. 48, 6973 (2009).

${ }^{6}$ G. A. Rakuljic and V. Leyva, Opt. Lett. 18, 459 (1993).

${ }^{7}$ C. L. Schoen, S. K. Sharma, C. E. Helsley, and H. Owen, Appl. Spectrosc. 47, 305 (1993).

${ }^{8}$ C. Moser and F. Havermeyer, Appl. Phys. B 95, 597 (2009).

${ }^{9}$ C. Xie, M. A. Dinno, and Y. Li, Opt. Lett. 27, 249 (2002).

${ }^{10}$ M. M. Carrabba, K. M. Spencer, C. Rich, and D. Rauh, Appl. Spectrosc. 44, 1558 (1990).

${ }^{11}$ M. J. Pelletier and R. C. Reeder, Appl. Spectrosc. 45, 765 (1991).

${ }^{12}$ B. Karsten, F. Havermeyer, L. Wenhai, M. Christophe, and D. Psaltis, "Holographic filtres," in Photorefractive Materials and Their Applications 3, edited by P. Günter and J.-P. Huignard (Springer, Berlin, 2007), pp. 295-319.

${ }^{13}$ G. T. Sincerbox, Current Trends in Optics, edited by J. C. Dainty (Academic, London, 1994), Chap. 14, Vol. 2.

${ }^{14}$ R. A. Lessard and G. Manivannan, Proc. SPIE 2405, 2 (1995).

${ }^{15}$ P. Cheben, T. Belenguer, A. Nuñez, F. del Monte, and D. Levy, Opt. Lett. 21, 1857 (1996)

${ }^{16}$ P. Cheben and M. L. Calvo, Appl. Phys. Lett. 78, 1490 (2001).

${ }^{17}$ F. Del Monte, O. Martínez-Matos, J. A. Rodrigo, M. L. Calvo, and P. Cheben, Adv. Mater. 18, 2014 (2006).

${ }^{18}$ K. Omura and Y. Tomita, J. Appl. Phys. 107, 023107 (2010).

${ }^{19}$ M. P. Hernández-Garay, O. Martínez-Matos, J. G. Izquierdo, M. L. Calvo, P. Vaveliuk, P. Cheben, and L. Bañares, Opt. Express 19, 1516 (2011).

${ }^{20}$ M. Haw, Nature 422, 556 (2003).

${ }^{21}$ D. Psaltis and F. Mok, Sci. Am. 273, 70 (1995).

${ }^{22}$ F. Mok, G. Zhou, and D. Psaltis, "Holographic read-only memory," in Holographic Data Storage, edited by H. J. Coufal, D. Psaltis, and G. T. Sincerbox (Springer, Berlin, 2000), pp. 399-407.

${ }^{23}$ H. Kogelnik, Bell Syst. Tech. J. 48, 2909 (1969).

${ }^{24}$ O. Martínez-Matos, J. A. Rodrigo, M. L. Calvo, V. Hevia-Martín, and P. Cheben, Opt. Mem. Neural Networks 18, 21 (2009).

${ }^{25}$ O. Martínez-Matos, M. L. Calvo, J. A. Rodrigo, P. Cheben, and F. del Monte, Appl. Phys. Lett. 91, 14115 (2007).

${ }^{26}$ A. V. Velasco, M. P. Hernández-Garay, M. L. Calvo, P. Cheben, and F. Del Monte, J. Appl. Phys. 109, 053106 (2011).

${ }^{27}$ G. Ramos, A. Alvarez-Herrero, T. Belenguer, F. del Monte, and D. Levy, Appl. Opt. 43, 4018 (2004). 